\title{
Anatomía de anillos de crecimiento de 80 especies arbóreas potenciales para estudios dendrocronológicos en la Selva Central, Perú
}

\author{
Lizandro Adal Beltrán Gutiérrez \& Gina Mariela Valencia Ramos \\ Laboratorio de tecnología de la madera, Facultad de Ciencias Forestales y del Ambiente, Universidad Nacional del \\ Centro del Perú, Huancayo, Perú; lizandro40bg@hotmail.com, iraya_21@hotmail.com
}

Recibido 09-I-2012. Corregido 20-X-2012. Aceptado 23-XI-2012.

\begin{abstract}
Anatomic characterization of growth-rings in $\mathbf{8 0}$ potential tree species for dendrocronological studies in the Central forest, Perú. The knowledge about the existence of annual tree rings in tropical trees, which was already found at the beginning of the last century, was ignored by many scientists for a long time. Wood samples of 80 tree species from seven different sites belonging to Satipo and Chanchamayo provinces in Central Forest, Perú. Wood slices were taken at 1.30m height, following the Peruvian Technical Norms (NTP) 251-008, COPANT norms 30:1-019 and IAWA (1989). Results showed that 24 of the 80 tree species analyzed showed a potential for dendrocronological studies, 25 had problems for growth-rings analysis, and 31 did not have potential. The problems most frequently found were: barely visible or irregular ring growth, parenchyma bands and multiseriate rays difficult to be identified in rings growth. The "T" Student test showed that the significant variation in vessel and fiber diameters between growth zones (Early-wood and late-wood) of species with potential for dendrocronology, do have a periodic cells production, so is possible to suggest the annual formation of each growth-ring. However, those species without potential to dendrocronology may be influenced by of a lot of factors, such as biotic and abiotic conditions of environment, as well as the genetic aspect of each species. Rev. Biol. Trop. 61 (3): 1025-1037. Epub 2013 September 01.
\end{abstract}

Key words: anatomic characterization, growth-rings, early wood, late wood, dendrocronology.

En el manejo forestal el conocimiento de la edad de los árboles es importante para determinar ciclos de corta y el tiempo durante el cual se renueva una masa forestal (Mendoza 1993). Una forma de calcular la edad en árboles tropicales es por conteo directo de los anillos de crecimiento; sin embargo, es necesario reconocer su existencia o que sean visibles en las especies de interés. La madera que se forma al inicio del anillo es denominada madera temprana y en ella las células son de paredes delgadas y lúmenes amplios. Por su parte, la madera formada al término del periodo vegetativo, tiene células de paredes anchas y lúmenes estrechos. Esta última zona recibe el nombre de madera tardía (Diaz 2003). En bosques templados dichos anillos están bien definidos por células de mayor tamaño en la madera temprana y de menor tamaño en la madera tardía. En contraste, en maderas tropicales la delimitación del anillo puede estar dada por estructuras celulares como parénquima marginal y fibras de paredes más gruesas o aplanadas en dirección radial. Por tanto, para confirmar la presencia de anillos se hacen estudios anatómicos microscópicos de los componentes celulares en la madera (elementos de vaso y fibras) (Carlquist 1988). El conocimiento acerca de la existencia de los anillos anuales en los árboles tropicales, fue encontrado a principios del siglo pasado e ignorado por muchos científicos a lo largo del tiempo (Worbes 1989). El análisis de los anillos de árboles tropicales es más difícil que el de las zonas templadas y boreales, debido a la compleja estructura anatómica de los árboles tropicales, condiciones de sitio, especies que 
forman más de un anillo por año (periodicidad), escasa información sobre la ecología del crecimiento para muchas especies, entre otros aspectos. Estos son factores que han dificultado el avance de estudios dendrocronológicos en los trópicos. Sin embargo, diferentes estudios demostraron la existencia de anillos anuales de crecimiento en muchas especies de árboles tropicales (Tomazello et al. 2004) y subtropicales (Navar et al. 2001). Esto debido a que muchos de los climas tropicales tienen estaciones predecibles con un exceso o escasez de disponibilidad de agua, cambios estacionales en la temperatura o una combinación de ambos, lo que origina la formación de los anillos de crecimiento (López et al. 2002).

En el Perú los estudios dendrocronológicos están fuertemente limitados por la dificultad de hallar especies potenciales para estudios dendrocronológicos, con anillos de crecimiento claramente distinguibles, por ello en pocas ocasiones se han realizado estudios dirigidos a establecer un método de recuento de anillos en estas especies.
La anatomía de la madera es importante ya que nos permite identificar las características necesarias que debe tener una especie para la dendrocronología así como también determinar la correcta identificación de los anillos de crecimiento. Esta investigación, nos ayudará a contribuir con información base para futuras investigaciones en el campo de la dendrocronología con especies tropicales que poco se ha estudiado en el Perú. El presente estudio tiene como objetivo caracterizar anatómicamente los anillos de crecimiento de 80 especies arbóreas potenciales para estudios dendrocronológicos en la Selva Central del Perú.

\section{MATERIALES Y MÉTODOS}

Zonas de estudio: Este estudio se llevó a cabo en las provincias de Chanchamayo y Satipo, Perú (Fig. 1). La precipitación total anual en la provincia de Satipo es de $2324.8 \mathrm{~mm}$, presentándose la mínima con $67.7 \mathrm{~mm}$ en el mes de julio y la máxima con $383.3 \mathrm{~mm}$ durante el mes de enero (Fig. 2). La temperatura
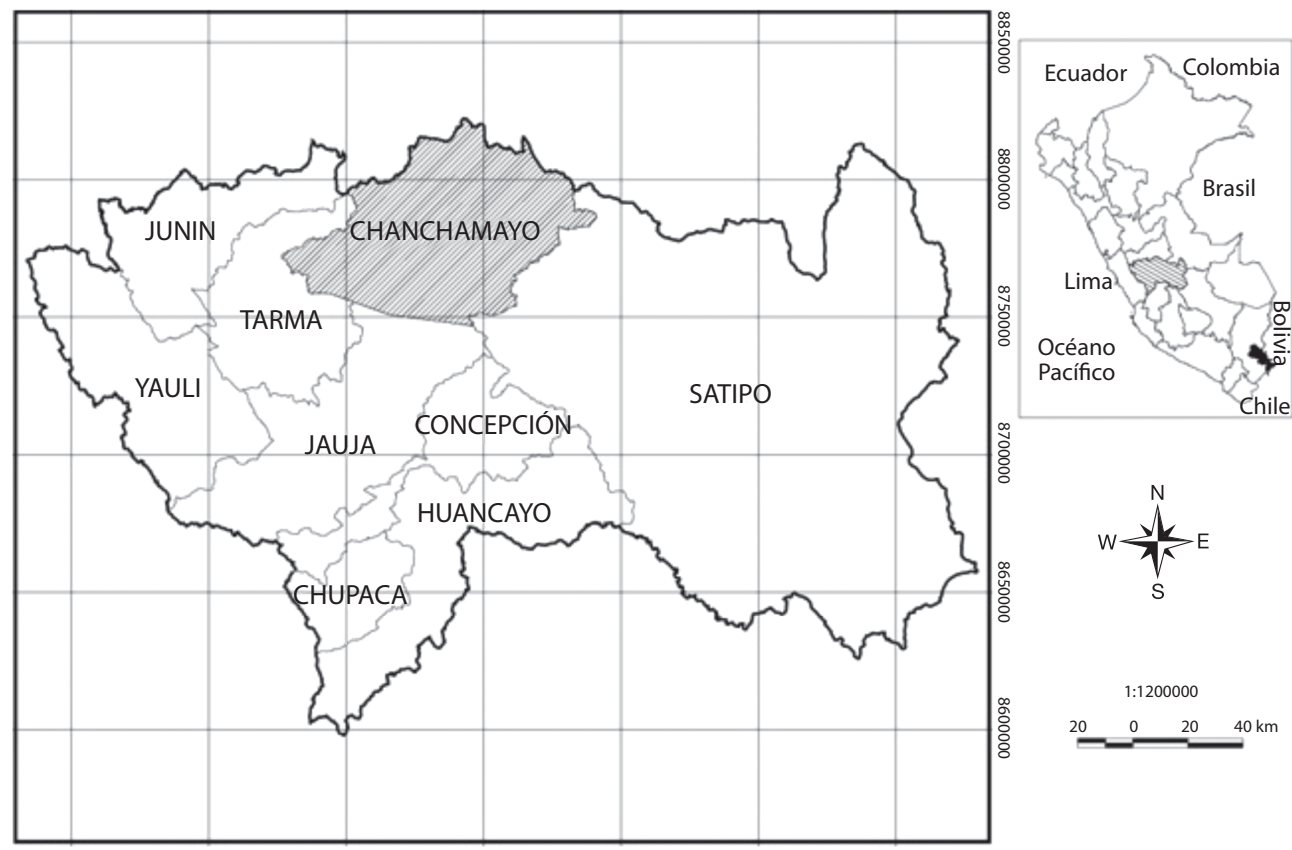

Fig. 1. Zonas de estudio. / Fig. 1. Study zones location. 


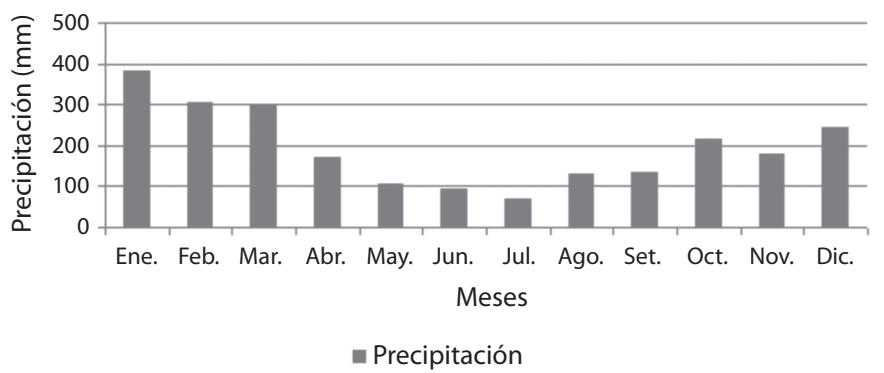

Fig. 2. Distribución de la precipitación (mm), 1980-2008. / Fig. 2. Rain fall distribution (mm), 1980-2008.

media durante el año es homogénea variando entre $24^{\circ} \mathrm{C}-27^{\circ} \mathrm{C}$ (Instituto de investigaciones de la Amazonia Peruana, 2011). La provincia de Chanchamayo tiene una precipitación total anual que varía entre $1500-3000 \mathrm{~mm}$, una temperatura que oscila entre $15-19^{\circ} \mathrm{C}$ (Young \& León 1999).

Rodajas de madera de 80 especies forestales se recolectaron de 28 familias en las zonas descritas en el cuadro 1.

Se seleccionaron de dos a tres árboles por especie de los cuales se obtuvieron rodajas de madera con una motosierra marca STIHL modelo B 175.1-2 000; a 1.30m sobre el suelo.
Se consideraron árboles de la categoría diamétrica que fluctúa entre $40-120 \mathrm{~cm}$ y de buena sanidad física y biológica. La recolección de muestras empleó la norma NTP (Normas Técnicas Peruanas) No251-008. Las características macroscópicas se observaron con una lente de mano de 10X, según la norma COPANT No30:1-019.

Con el fin de observar la presencia de los anillos de crecimiento visibles, primero se obtuvieron tiras de madera de $2 \mathrm{~cm}$ de lado, que fueron pulidas con lijas de diferente granulometría 80, 60, 100,120, 220, 240 y 360. Se emplearon sustancias contrastantes como:

\section{CUADRO 1}

Sitios de recolección de las muestras

TABLE 1

Zones where samples were taken

\begin{tabular}{|c|c|c|c|c|}
\hline \multirow{2}{*}{ Comunidad Nativa } & \multicolumn{2}{|c|}{ Ubicación } & \multirow{2}{*}{ Ubicación Geográfica } & \multirow{2}{*}{ Altitud (msnm) } \\
\hline & Provincia & Distrito & & \\
\hline San Juan de Mazaronquiari & Satipo & $\begin{array}{l}\text { San Martín } \\
\text { de Pangoa }\end{array}$ & $11^{\circ} 38^{\prime} 49^{\prime \prime} \mathrm{S}-74^{\circ} 20^{\prime} 26^{\prime \prime} \mathrm{W}$ & $1109-1246$ \\
\hline Tres Unidos de Matereni & Satipo & $\begin{array}{l}\text { San Martín } \\
\text { de Pangoa }\end{array}$ & $11^{\circ} 43^{\prime} 21^{\prime \prime} \mathrm{S}-74^{\circ} 14^{\prime} 0^{\prime \prime} \mathrm{W}$ & $980-1000$ \\
\hline Matzuriniari & Satipo & $\begin{array}{l}\text { San Martín } \\
\text { de Pangoa }\end{array}$ & $11^{\circ} 26^{\prime} 20^{\prime \prime} \mathrm{S}-74^{\circ} 18^{\prime} 1^{\prime \prime} \mathrm{W}$ & 1500 \\
\hline Oviri & Satipo & Río Tambo & $11^{\circ} 18^{\prime} 33^{\prime \prime} \mathrm{S}-73^{\circ} 49^{\prime} 24^{\prime \prime} \mathrm{W}$ & $400-760$ \\
\hline Río Blanco Chiriteni & Satipo & $\begin{array}{l}\text { San Martín } \\
\text { de Pangoa }\end{array}$ & $11^{\circ} 45^{\prime} 37^{\prime \prime} \mathrm{S}-74^{\circ} 22^{\prime} 37^{\prime \prime} \mathrm{W}$ & $1650-1800$ \\
\hline Centro Potzoteni & Satipo & $\begin{array}{l}\text { San Martín } \\
\text { de Pangoa }\end{array}$ & $11^{\circ} 22^{\prime} 55^{\prime \prime} \mathrm{S}-74^{\circ} 14^{\prime} 7^{\prime \prime} \mathrm{W}$ & $1036-1098$ \\
\hline Zona Vitoc & Chanchamayo & Vitoc & $11^{\circ} 13^{\prime} 33^{\prime \prime} \mathrm{S}-75^{\circ} 20^{\prime} 44^{\prime \prime} \mathrm{W}$ & $1100-1800$ \\
\hline
\end{tabular}


safranina al 1\%, limón, kerosene, ron de quemar y thinner para mejorar la visibilidad de los anillos de crecimiento.

Para confirmar la presencia de anillos de crecimiento se hacen estudios anatómicos microscópicos de los componentes celulares en la madera (elementos de vaso y fibras). Las variables consideradas fueron las siguientes: anillos de crecimiento, diámetro de vasos y diámetro de fibras. Se empleó el analizador de imágenes Leica Aplication Suite EZ (Versión 1.6.0, 2008), adaptado a una cámara de video Modelo DC 150 7.1, y a un microscopio trinocular Leica, para efectuar las observaciones y mediciones. En el laboratorio las muestras se seccionaron en probetas de $2 \times 2 \times 2 \mathrm{~cm}$ de lado, se hirvieron en agua por un lapso de $12-40 \mathrm{~h}$ dependiendo de la dureza de la especie. Posteriormente, se procedió a ablandar las muestras con alcohol al 30\% y glicerina al 70\%. Con un micrótomo de deslizamiento horizontal (modelo SM 2000R), se obtuvieron cortes transversales de un espesor entre $20-30 \mu \mathrm{m}$, dependiendo de la especie. Las láminas se deshidrataron en diferentes concentraciones de alcohol (30\%, $70 \%, 80 \%$ y $95 \%$ ) por un lapso de $20 \mathrm{~min}$, se aclararon con el reactivo verde brillante y se colorearon con safranina al 1\%, luego se colocaron en un portaobjeto con una a dos gotas de bálsamo de Canadá, y finalmente se cubrieron con una lámina cubreobjetos.

De las especies que presentaron potencial para estudios dendrocronológicos debido a que tienen características importantes como: anillos distintos (visibles), anillos regulares; no presentando dificultades en el tipo de parénquima axial (bandas anchas de parénquima), tipo de transición gradual de zonas fibrosas y/o traqueidas, radios multiseriados; que dificulten la identificación de los anillos de crecimiento; se astilló la madera temprana y madera tardía semejante a los palitos de fósforo, de aproximadamente $1.5 \mathrm{~mm}$ de espesor con la ayuda de una cuchilla, que se obtuvieron a partir de los cubos de $2 \times 2 \times 2 \mathrm{~cm}$. Para la maceración de las astillas estas se colocaron en un frasco con tapa hermética con una solución de ácido acético y peróxido de hidrógeno en proporciones de uno a dos, respectivamente, de manera que cubrió completamente las astillas; se secaron a $65^{\circ} \mathrm{C}$ por 48-72h. Además, se realizaron 50 mediciones del diámetro de vasos y de fibras de cada repetición (25 en madera temprana y 25 en madera tardía). Para determinar si existe diferencia estadística significativa entre la madera temprana y tardía, se empleó la estadística inferencial, prueba de " $\mathrm{T}$ " de Student al 95\% de intervalo de confianza, para muestras independientes. Estos análisis estadísticos se realizaron con el software XLSTAT.

\section{RESULTADOS}

La caracterización anatómica de los anillos de crecimiento de las especies analizadas se describe a continuación en orden por familia (Fig. 3, Cuadro 4).

Anacardiaceae: Fueron investigadas dos especies de esta familia: Spondias mombin L., presenta anillos de crecimiento distintos a simple vista, regulares, delimitados por una banda de fibras acortadas radialmente, con potencial dendrocronológico; Astronium lecoentei Ducke. velsp. aff tiene anillos de crecimiento poco distintos, regulares, delimitados por una banda de fibras acortadas radialmente, con dificultad para estudios dendrocronológicos.

Apocynaceae: La especie Aspidosperma parvifolium A. DC. presentó anillos de crecimiento con potencial dendrocronológico, limitados por una banda de fibras acortadas radialmente.

Bignoniaceae: La especie Jacaranda copaia (Aubl.) D. Don. presentó anillos de crecimiento distintos a simple vista, regulares, delimitados por una banda de fibras acortadas radialmente, con potencial dendrocronológico.

Caryocaraceae: Fueron investigadas dos especies de esta familia: Caryocar amigdaliforme Ruiz \& Pav. ex G. Don. presentó anillos de crecimiento distintos a simple vista, regulares, delimitados por una banda de fibras 

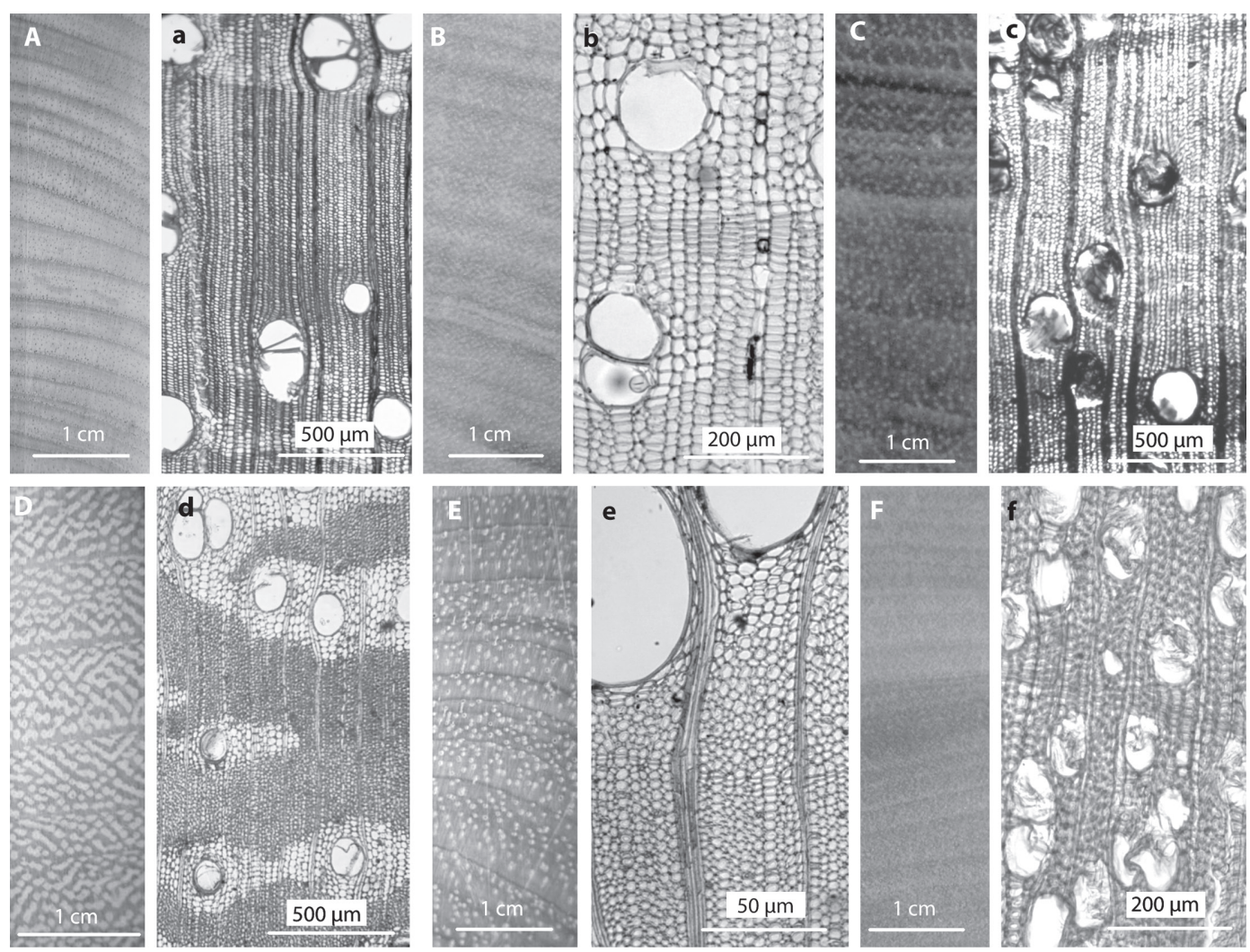

Fig. 3. A) Vista macroscópica de Cedrela odorata L. (a) Límites de anillos a nivel microscópico de Cedrela odorata L. (B) Vista macroscópica de Aniba panurensis (Meisn.) Mez. (b) Tejido de fibras acortadas radialmente de Aniba panurensis (Meisn.). (C) Vista macroscópica de Juglans neotropica Diels. (c) Porosidad semicircular en Juglans neotropica Diels. (D) Vista macroscópica de Amburana cearensis (Allemao) A.C.Sm. (d) Limite del anillo definido por transición abrupta de zonas fibrosas en Amburana cearensis (Allemao) A.C.Sm. (E) Vista macroscópica de Cedrelinga cateniformis (Ducke) Ducke. (e) Límite del anillo definido por transición abrupta de zonas fibrosas en Cedrelinga cateniformis (Ducke) Ducke. (F) Vista macroscópica de Aspidosperma parvifolium A. DC. (f) Límite del anillo definido tejido de fibras acortadas radialmente en Aspidosperma parvifolium A. DC.

Fig. 3. (A) Macroscopic view of Cedrela odorata L. (a) Mircoscopic Growth-limits of Cedrela odorata L. (B) Macroscopic view of Aniba panurensis (Meisn.) Mez. (b) Fibre tissue radially flattened in Aniba panurensis (Meisn.). (C) Macroscopic view of Juglans neotropica Diels. (c) Semi-ring-porous in Juglans neotropica Diels. (D) Macroscopic view of Amburana cearensis (Allemao) A.C.Sm. (d) Growth-ring limit defined for several transitions of fibre zones in Amburana cearensis (Allemao) A.C.Sm. (E) Macroscopic view of Cedrelinga cateniformis (Ducke) Ducke. (e) Growth-ring limit defined for several transitions of fibre zones in Cedrelinga cateniformis (Ducke) Ducke. (F) Macroscopic view of Aspidosperma parvifolium A. DC. (f) Growth-ring limit defined for several rows of fiber cells radially flattened in Aspidosperma parvifolium A. DC.

acortadas radialmente, con potencial dendrocronológico; Anthodiscus peruanus Baill, tiene anillos de crecimiento poco distintos, regulares, delimitados por una banda de fibras acortadas radialmente, con dificultad para estudios dendrocronológicos.

Clusiaceae: La especie Calophyllum brasiliense Cambess. presentó anillos de crecimiento poco distintos (visibles), delimitados por una banda de fibras acortadas radialmente, con dificultad para dendrocronología.

\section{Combretaceae: Terminalia oblonga (Ruiz} \& Pavon) Steud. presentó anillos de crecimiento distintos a simple vista, regulares, delimitados por una banda de fibras acortadas radialmente, con potencial dendrocronológico. 
Buchenavia amazonia Alwan \& Stace., presentó anillos de crecimiento poco distintos, irregulares, delimitados por una línea delgada de parénquima marginal, con dificultad para estudios dendrocronológicos.

Euphorbiaceae: De las tres especies estudiadas de esta familia dos especies tienen potencial dendrocronológico: Hevea brasiliensis (Willd. ex A. Juss.) Müll. Arg. y Hura crepitans L. presentaron anillos de crecimiento distintos a simple vista, regulares delimitados por una banda de tejido de fibras acortadas radialmente.

Fabaceae: Se estudiaron 13 especies de esta familia, de las cuales reportamos cinco especies con potencial dendrocronológico. Amburana cearensis (Allemao) A.C.Sm., Cedrelinga cateniformis (Ducke) Ducke y Enterolobium schomburgkii (Benth.) Benth, las cuales presentaron anillos de crecimiento distintos, regulares, delimitados por una banda de fibras acortadas radialmente. Copaifera paupera (Herzog) Dwyer., presenta anillos de crecimiento distintos a simple vista, regulares, delimitados por una banda de parénquima marginal y Schizolobium amazonicum Huber ex Ducke. presenta anillos de crecimientos delimitados por una banda de fibras acortadas radialmente asociado a una fina banda de parénquima marginal. Por otro lado, tres especies tienen dificultad para estudios dendrocronológicos: Tachigali chrysaloides Van der Werff y Myroxylon peruiferum Lf. tienen anillos de crecimiento poco distintos, regulares, delimitados por una banda de fibras acortadas radialmente, Platymiscium pinnatum (Jacq.) Dugand., tiene anillos de crecimiento poco distintos (debido al color del duramen) regulares, delimitados por una banda de parénquima marginal.

Juglandaceae: La única especie estudiada de esta familia es Juglans neotropica Diels, la cual tiene potencial para dendrocronología, presentó anillos de crecimiento delimitados por la diferencia en el diámetro de los poros (porosidad semicircular), asociado a una banda delgada de parénquima marginal.

Lauraceae: De las 12 especies investigadas de esta familia, tres especies mostraron potencial para dendrocronología: Ocotea aciphylla (Nees) Mez, Aniba panurensis (Meisn.) Mezy y Nectandra membranacea (Sw.) Griseb \& Rohwer, las cuales presentaron anillos de crecimiento delimitados por una banda de tejido de fibras acortadas radialmente. Así mismo dos especies de esta familia presentan dificultad para dendrocronología: Persea areolatocostae (C.K. Allen) van y Ocotea gracilis (Meisn.) Mez. tienen anillos de crecimiento poco distintos, regulares, delimitados por una banda delgada de fibras acortadas radialmente.

Lecythidaceae: Cariniana decandra Ducke. presentó anillos de crecimiento distintos a simple vista, regulares, delimitados por una banda de fibras acortadas radialmente, con potencial para dendrocronología.

Malvaceae: Se estudiaron cinco especies de esta familia de las cuales Matisia bicolor Ducke y Guazuma crinita Mart. mostraron potencial para dendrocronología, presentando anillos de crecimiento distintos, regulares, delimitados por una banda de fibras acortadas radialmente. Por otro lado Ceiba samauma (Mart.) J.k. Schum. mostró anillos de crecimiento poco distintos, delimitados por una banda delgada de parénquima marginal, con dificultad para estudios dendrocronológicos.

Meliceae: Fueron investigadas tres especies de esta familia de las cuales solo Cedrela odorata L. mostró potencial dendrocronológico, esta especie presentó anillos de crecimiento distintos a simple vista, regulares, caracterizados por tener porosidad de circular a semicircular y asociados a una banda de parénquima marginal.

Moraceae: Se estudiaron nueve especies de esta familia, se reportan tres especies potenciales para dendrocronología: Brosimum 
alicastrum Sw. y Brosimum multinervium C.C. Berg que presentaron anillos distintos delimitados por una banda de fibras acortadas radialmente y Pseudolmedia rigida (K1. \& Karst.) con anillos delimitados por una banda de fibras acortadas radialmente, así mismo, se observó una decreciente frecuencia de bandas de parénquima desde el inicio hacia el final del anillo de crecimiento.

Myristicaceae: Las seis especies estudiadas de esta familia presentaron anillos de crecimiento con dificultad para dendrocronología, Otoba parvifolia (Markgr.) A. H. Gentry, Otoba glycicarpa (Ducke) W. Rodríguez \& T.S. Jaramillo, Virola duckei A.C. S.M., Virola flexuosa A.C. Sm., al mostrar anillos de crecimiento poco distintos, regulares, delimitados por una banda de fibras acortadas radialmente.

Rosaceae: Prunus detrita J.F. Macbr. mostró anillos de crecimiento poco distintos, regulares, delimitados por una banda de fibras acortadas radialmente.

Rubiaceae: Se estudiaron tres especies de esta familia, de las cuales solo la especie Calycophyllum spruceaunum (Benth.) Hookf. Ex Schum tiene potencial para dendrocronología, con anillos delimitados por una banda de fibras acortadas radialmente. Así mismo Calycophyllum megistocaulum (K. Krause) C.M. Taylor, tienen dificultad para estudios dendrocronológicos, con anillos de crecimiento poco distintos, regulares, delimitados por una banda de fibras acortadas radialmente.

Staphyleaceae: Huertea glandulosa (Ruiz \& Pavon) Steud. presentó anillos de crecimiento poco distintos, debido al color de la madera (duramen), regulares, delimitados por una banda de fibra acortadas radialmente, con dificultad para dendrocronología.

Theaceae: Gordonia fruticosa (Schrad.) H. Keng. presentó anillos de crecimiento poco distintos, regulares, delimitados por una banda de fibra de mayor espesor de pared acortados radialmente, con potencial dendrocronológico con dificultad.

Vochysiaceae: Vochysia vismifolia Spruce \& Warm. mostró anillos de crecimiento poco distintos, regulares, delimitados por una banda de fibra acortadas radialmente, con dificultad para dendrocronología.

La prueba de " $T$ " de Student realizada a las 23 especies que presentaron buen potencial para dendrocronología, concluyó que 10 especies mostraron diferencia estadística significativa en el diámetro de vasos de la madera temprana frente a la madera tardía: Pseudolmedia rigida y Copaifera paupera, presentó diferencia estadística significativa $(\mathrm{p}<0.05)$; así mismo Enterolobium schomburgkii Schizolobium amazonicum, Juglans neotropica, Cedrela odorata, Brosimum alicastrum, Amburana cearensis, Spondias mombin y Terminalia oblonga, presentaron diferencias estadísticas altamente significativas $(\mathrm{p}<0.01)($ Cuadro 2$)$. Con respecto al diámetro de fibras, 11 especies mostraron diferencias estadísticas significativas entre madera temprana y tardía: Juglans neotropica y Brosimum alicastrum presentaron diferencia estadística significativa $(\mathrm{p}<0.05)$, así mismo Schizolobium amazonicum, Aniba panurensis, Nectandra membranacea, Matisia bicolor, Hura crepitans, Amburana cearensis, Jacaranda copaia, Spondias mombin y Cedrelinga cateniformis, reportaron diferencias altamente significativas $(\mathrm{p}<0.01)$ (Cuadro 3$)$.

\section{DISCUSIÓN}

En relación a la porosidad, 76 especies presentaron poros difusos, Cedrela odorata L. posee porosidad de circular a semicircular, Juglans neotropica Diels y Schizolobium amazonicum Huber ex Ducke, tienen porosidad semicircular y Retrophyllum rospigliosii (Pilg) C.N. Page, no poseen poros por ser una conífera. Así también, Campos (2009), reportó veinticinco especies con porosidad difusa y Cedrela odorata con porosidad semicircular, con estudios similares realizados en Brasil y Perú. Esta característica de la porosidad difusa 
CUADRO 2

Prueba de "T" Student para los diámetros tangenciales de vasos

TABLE 2

"T" Test of vessel diameters

\begin{tabular}{|c|c|c|c|c|c|c|}
\hline \multirow{2}{*}{ Especie } & \multirow{2}{*}{$n$} & \multicolumn{2}{|c|}{ Madera temprana } & \multicolumn{2}{|c|}{ Madera Tardía } & \multirow{2}{*}{ P-Valor } \\
\hline & & Promedio & $\mathrm{S}$ & Promedio & $\mathrm{S}$ & \\
\hline Aspidosperma parvifolium A. DC & 50 & 102.409 & 26.544 & 95.733 & 16.086 & 0.288 \\
\hline Hevea brasiliensis (Willd. ex A. Juss.) Mu:1l.Arg. & 50 & 184.276 & 43.526 & 176.711 & 40.108 & 0.526 \\
\hline Enterolobium Schomburgkii (Benth.) Benth. & 50 & 171.162 & 31.476 & 88.118 & 22.433 & $<0.0001 * *$ \\
\hline Schizolobium amazonicum Huber ex Ducke & 50 & 269.900 & 51.784 & 173.552 & 63.258 & $<0.0001 * *$ \\
\hline Juglans neotropica Diels & 50 & 278.889 & 66.786 & 183.384 & 61.004 & $<0.0001 * *$ \\
\hline Aniba panurensis (Meisn.) Mez. & 50 & 174.735 & 31.508 & 171.762 & 31.633 & 0.741 \\
\hline Nectandra membranacea (Sw.) Griseb \& Rohwer & 50 & 180.306 & 28.506 & 165.343 & 90.347 & 0.434 \\
\hline Matisia bicolor Ducke & 50 & 272.556 & 68.241 & 257.798 & 50.813 & 0.390 \\
\hline Brosimum multinervium C.C. Berg & 50 & 203.522 & 39.919 & 183.744 & 40.483 & 0.088 \\
\hline Pseudolmedia rigida (K1. \& Karst.) Cuatr. & 50 & 173.208 & 28.574 & 144.644 & 31.821 & $0.002 *$ \\
\hline Calycophyllum spruceaunum (benth.) Hookf.exSchum. & 50 & 97.674 & 16.389 & 95.109 & 13.530 & 0.549 \\
\hline Caryocar amigdaliforme Ruiz \& Pav. ex G. Don & 50 & 182.950 & 37.920 & 171.005 & 38.518 & 0.121 \\
\hline Cariniana decandra Ducke & 50 & 196.448 & 59.793 & 173.109 & 64.326 & 0.063 \\
\hline Hura crepitans L. & 50 & 148.862 & 37.603 & 142.461 & 28.515 & 0.340 \\
\hline Cedrela odorata L. & 50 & 306.567 & 71.917 & 178.428 & 43.069 & $<0.0001 * *$ \\
\hline Brosimum alicastrum $\mathrm{Sw}$. & 50 & 166.318 & 43.915 & 145.197 & 27.143 & $0.005 * *$ \\
\hline Copaifera paupera (Herzog) Dwyer & 50 & 207.405 & 55.866 & 173.219 & 34.264 & $0.012 *$ \\
\hline Amburana cearensis (Allemao) A.C.Sm. & 50 & 174.208 & 29.994 & 92.080 & 18.301 & $<0.0001 * *$ \\
\hline Ocotea aciphylla (Nees) Mez. & 50 & 207.088 & 93.529 & 199.231 & 56.443 & 0.612 \\
\hline Jacaranda copaia (Aubl.) D.Don & 50 & 261.786 & 56.952 & 247.895 & 61.454 & 0.244 \\
\hline Spondias mombin L. & 50 & 229.656 & 39.528 & 175.082 & 42.281 & $<0.0001 * *$ \\
\hline Cedrelinga cateniformis (Ducke) Ducke & 50 & 408.506 & 107.561 & 386.295 & 131.597 & 0.358 \\
\hline Terminalia oblonga (Ruiz \& Pavon) Steud. & 50 & 276.688 & 51.498 & 132.871 & 30.277 & $<0.0001 * *$ \\
\hline
\end{tabular}

n: Número de mediciones. S: Desviación estándar. Fuente: Elaborado con XLSTAT.

(*): Significativo al 95\% de confianza (P-Valor $<0.05$ )

$(* *)$ : Altamente significativo al 99\% de confianza $(\mathrm{P}-$ Valor $<0.01)$

es corroborada por el (IAWA 1989 y Chavesta 2006), quienes reportan este tipo de porosidad en la extensa mayoría de las especies tropicales. Así mismo las condiciones ambientales que presenta la zona de estudio sería fundamental para la presencia de la porosidad difusa, donde el crecimiento está determinado por la precipitación, siendo esta de manera continua casi en todo el año, con una sola época de lluvias escasas (mayo-setiembre).

De las 23 especies que mostraron anillos de crecimiento distintos (visibles), 20 especies, están delimitadas por una banda de tejido fibroso acortado radialmente, concordando con Díaz
(2003), quien menciona que el acortamiento de fibras y traqueidas está presente en la mayoría de las especies de latifoliadas. Esto se debería a que al final de la estación de crecimiento, los factores tales como sequía, reducen la concentración de auxinas y por lo tanto fomentan producción de células de diámetro pequeño; mientras tanto, la acumulación de inhibidores de crecimiento causa reducción en el ritmo de producción de nuevas células, cesando a su vez el crecimiento de nuevos brotes y desarrollo de nuevas hojas. Por lo tanto, el producto fotosintético producido por las hojas desarrolladas a plenitud, está disponible para la síntesis de 
CUADRO 3

Diámetros promedio de vasos y fibras

TABLE 3

Average diameters vessel and fibers

\begin{tabular}{|c|c|c|c|}
\hline \multirow{2}{*}{ Especie } & \multirow{2}{*}{$n$} & \multicolumn{2}{|c|}{ Diametro promedio } \\
\hline & & Vasos $(\mu \mathrm{m})$ & Fibras $(\mu \mathrm{m})$ \\
\hline Aspidosperma parvifolium A. DC & 50 & 99.071 & 26.233 \\
\hline Hevea brasiliensis (Willd. ex A. Juss.) Mu:ll.Arg. & 50 & 180.493 & 23.034 \\
\hline Enterolobium schomburgkii (Benth.) Benth. & 50 & 129.640 & 23.604 \\
\hline Schizolobium amazonicum Huber ex Ducke & 50 & 221.726 & 29.581 \\
\hline Juglans neotropica Diels & 50 & 231.136 & 25.948 \\
\hline Aniba panurensis (Meisn.) Mez & 50 & 173.249 & 29.975 \\
\hline Nectandra membranacea (Sw.) Griseb \& Rohwer & 50 & 172.825 & 24.980 \\
\hline Matisia bicolor Ducke & 50 & 265.177 & 41.954 \\
\hline Brosimum multinervium C.C. Berg & 50 & 193.633 & 28.514 \\
\hline Pseudolmedia rigida (K1. \& Karst.) Cuatr. & 50 & 158.926 & 18.469 \\
\hline Calycophyllum spruceaunum (benth.) Hookf.ex Schum. & 50 & 96.392 & 23.379 \\
\hline Caryocar amigdaliforme Ruiz \& Pav. ex G. Don & 50 & 176.977 & 30.491 \\
\hline Cariniana decandra Ducke & 50 & 184.779 & 19.387 \\
\hline Hura crepitans $\mathrm{L}$. & 50 & 145.661 & 30.611 \\
\hline Cedrela odorata $\mathrm{L}$. & 50 & 242.498 & 26.870 \\
\hline Brosimum alicastrum $\mathrm{Sw}$. & 50 & 155.758 & 17.928 \\
\hline Copaifera paupera (Herzog) Dwyer & 50 & 190.312 & 24.569 \\
\hline Amburana cearensis (Allemao) A.C.Sm. & 50 & 133.144 & 22.305 \\
\hline Ocotea aciphylla (Nees) Mez & 50 & 203.160 & 28.990 \\
\hline Jacaranda copaia (Aubl.) D.Don & 50 & 254.840 & 23.064 \\
\hline Spondias mombin L. & 50 & 202.369 & 32.589 \\
\hline Cedrelinga cateniformis (Ducke) Ducke & 50 & 397.400 & 29.238 \\
\hline Terminalia oblonga (Ruiz \& Pavon)Steud & 50 & 204.779 & 25.511 \\
\hline
\end{tabular}

$\mathrm{n}$ : Número de mediciones.

la pared celular. Posteriormente, el resultado será células de paredes gruesas y diámetros pequeños (Vera et al. 2011). Esto se refleja en las dimensiones de las células de madera tardía de paredes anchas y lúmenes estrechos y madera temprana de paredes delgadas y lúmenes amplios, estas dos partes del anillo, producen diferencias de tonalidad.

Las 12 especies de la familia Lauraceae, presentaron anillos de crecimiento delimitados por una banda de tejido fibroso acortados radialmente, lo que coincide con lo reportado por Tomazello et al. (2004) y con estudios similares realizados en Brasil, quienes mencionan que es común encontrar en especies de la familia Lauraceae este tipo de delimitación. El crecimiento radial en los árboles, originado por el cambium, genera tejido xilemático secundario y floema durante el periodo de crecimiento. Este crecimiento depende en gran medida de las características genéticas de cada especie y de la influencia de factores internos (edad) y externos (clima, suelo, competencia y sitio).

De las 13 especies de la familia Fabaceae, cuatro especies presentan anillos de crecimiento delimitados por una banda de parénquima marginal, contrario a lo hallado por (Tomazello et al. 2004) quienes mencionan que las bandas de parénquima marginal son comunes en esta familia. Estas características podría deberse a 
CUADRO 4

Anatomía de anillos de crecimiento y análisis del potencial dendrocronológico

TABLE 4

Characterize of Growth-Rings and dendrocronological potential analysis

\begin{tabular}{|c|c|c|c|c|c|c|c|}
\hline \multirow{2}{*}{ Familia } & \multirow{2}{*}{ Especie } & \multicolumn{5}{|c|}{ Características } & \multirow{2}{*}{ Dificultades encontradas } \\
\hline & & $\mathrm{D}$ & $\mathrm{P}$ & $\mathrm{TF}$ & PB & PD & \\
\hline Anacardiaceae & Astronium lecoentei Ducke. velsp. aff. & 2 & 3 & 2 & 3 & 2 & Transición gradual de zonas fibrosas \\
\hline Anacardiaceae & Spondias mombin L. & 1 & 3 & 1 & 3 & 1 & \\
\hline Apocinaceae & Aspidosperma parvifolium A. DC & 1 & 3 & 1 & 3 & 1 & \\
\hline Bignoniaceae & Jacaranda copaia (Aubl.) D. Don & 1 & 3 & 1 & 3 & 1 & \\
\hline Burseraceae & Tetragastris altissima (Aubl.) Sw. & 3 & 3 & 3 & 3 & 3 & Anillos irregulares, indistintos \\
\hline Caryocariaceae & Anthodiscus peruanus Baill. & 2 & 3 & 2 & 3 & 27 & Transición gradual de zonas fibrosas \\
\hline Caryocariaceae & Caryocar amigdaliforme Ruiz \& Pav. ex G. Don & 1 & 3 & 1 & 3 & 1 & \\
\hline Clusiaceae & Calophyllum brasiliense Cambess & 2 & 3 & 2 & 3 & 27 & Transición gradual de zonas fibrosas \\
\hline Combretaceae & Buchenavia amazonia Alwan \& Stace & 2 & 3 & 3 & 1 & 3 & Anillos irregulares, textura fina \\
\hline Combretaceae & Terminalia oblonga (Ruiz \& Pavon)Steud & 1 & 3 & 1 & 3 & 1 & \\
\hline Euforbiaceae & Hura crepitans $\mathrm{L}$. & 1 & 3 & 2 & 3 & 1 & \\
\hline Euforbiaceae & Tetrorchidium rubrivenium Poepp. Vel sp. aff. & 3 & 3 & 3 & 3 & 3 & Anillos indistintos (no visibles) \\
\hline Euforbiaceae & Hevea brasiliensis (Willd. ex A. Juss.) Mu:ll.Arg & 1 & 3 & 1 & 2 & 1 & \\
\hline Fabaceae & Amburana cearensis (Allemao) A.C.Sm. & 1 & 3 & 1 & 3 & 1 & \\
\hline Fabaceae & Cedrelinga cateniformis (Ducke) Ducke & 1 & 3 & 1 & 3 & 1 & \\
\hline Fabaceae & Copaifera paupera (Herzog) Dwyer & 1 & 3 & 3 & 1 & 1 & \\
\hline Fabaceae & Tachigali chrysaloides Van der Werff & 2 & 3 & 2 & 3 & 27 & Transición gradual de zonas fibrosas \\
\hline Fabaceae & Apuleia leiocarpa (Vogel) J.F. Macbr. & 2 & 3 & 3 & 1 & 3 & Anillos irregulares, textura fina \\
\hline Fabaceae & Albizia carbonaria Britton & 3 & 3 & 3 & 3 & 3 & Anillos indistintos (no visibles) \\
\hline Fabaceae & Myroxylon peruiferum Lf. & 2 & 3 & 2 & 3 & 27 & Transición gradual de zonas fibrosas \\
\hline Fabaceae & Platymiscium pinnatum (Jacq.) Dugand & 2 & 3 & 3 & 1 & 2 & $\begin{array}{l}\text { Anillos poco distintos, debido al color } \\
\text { de la madera }\end{array}$ \\
\hline Fabaceae & Diplotropis purpurea (Rich.) Amshoff & 3 & 3 & 3 & 3 & 3 & Anillos indistintos (no visibles) \\
\hline Fabaceae & Enterolobiums chomburgkii (Benth.) Benth. & 1 & 3 & 1 & 3 & 1 & \\
\hline Fabaceae & Inga cf. Acrocephala Standl. & 3 & 3 & 3 & 3 & 3 & $\begin{array}{l}\text { Anillos indistintos (poco visibles) e } \\
\text { irregulares }\end{array}$ \\
\hline Fabaceae & Myroxylon balsamum (K.) Harms & 2 & 3 & 2 & 3 & 2 & Anillos poco distintos en partes del leño \\
\hline Fabaceae & Schizolobium amazonicum Huber ex Ducke & 1 & 2 & 1 & 1 & 1 & \\
\hline Juglandaceae & Juglans neotropica Diels & 1 & 2 & 3 & 1 & 1 & \\
\hline Lauraceae & Ocotea aciphylla (Nees) Mez & 1 & 3 & 2 & 3 & 1 & \\
\hline Lauraceae & Persea areolatocostae (C.K. Allen) van & 2 & 3 & 2 & 3 & 27 & Transición gradual de zonas fibrosas \\
\hline Lauraceae & Aniba panurensis (Meisn.) Mez & 1 & 3 & 1 & 3 & 1 & \\
\hline Lauraceae & Caryodaphnosis fosterii Van der Werff & 2 & 3 & 2 & 3 & 27 & Transición gradual de zonas fibrosas \\
\hline Lauraceae & Nectandra membranacea (Sw.) Griseb \& Rohwer & 1 & 3 & 1 & 3 & 1 & \\
\hline Lauraceae & Ocotea argyrophylla Ducke & 3 & 3 & 3 & 3 & 3 & Anillos indistintos (no visibles) \\
\hline Lauraceae & Ocotea gracilis (Meisn.) Mez & 2 & 3 & 2 & 3 & 27 & Transición gradual de zonas fibrosas \\
\hline Lauraceae & Ocotea javitensis (Kunth) Pittier & 2 & 3 & 2 & 3 & 2 & Anillos poco distintos en partes del leño \\
\hline Lauraceae & Ocotea leucoxylon (Sw.) de Lanessan & 2 & 3 & 2 & 3 & 27 & Transición gradual de zonas fibrosas \\
\hline Lauraceae & Ocotea sp. & 2 & 3 & 2 & 3 & 27 & Transición gradual de zonas fibrosas \\
\hline Lauraceae & Pleurothyrium brochidodromum Van der Werff & 2 & 3 & 2 & 3 & $3 \mathrm{I}$ & Presenta anillos irregulares \\
\hline Lauraceae & Pleurothyrium cuneifolium Nees & 2 & 3 & 2 & 3 & 2 & Anillos poco distintos en la albura \\
\hline Lecythidaceae & Cariniana decandra Ducke & 1 & 3 & 1 & 3 & 1 & \\
\hline
\end{tabular}


CUADRO 4 (Continuación) / TABLE 4 (Continued)

\begin{tabular}{|c|c|c|c|c|c|c|c|}
\hline \multirow{2}{*}{ Familia } & \multirow{2}{*}{ Especie } & \multicolumn{5}{|c|}{ Características } & \multirow{2}{*}{ Dificultades encontradas } \\
\hline & & $\mathrm{D}$ & $\mathrm{P}$ & $\mathrm{TF}$ & $\mathrm{PB}$ & PD & \\
\hline Malvaceae & Ceiba samauma (Mart.) J.k. Schum. & 2 & 3 & 1 & 1 & 21 & $\begin{array}{l}\text { Radios multiseriados hasta de } 13 \text { células } \\
\text { de ancho }\end{array}$ \\
\hline Malvaceae & Guazuma crinita Mart. & 1 & 3 & 2 & 3 & 1 & \\
\hline Malvaceae & Heliocarpus americanus L. & 2 & 3 & 2 & 3 & 27 & Transición gradual de zonas fibrosas \\
\hline Malvaceae & Matisia bicolor Ducke & 1 & 3 & 1 & 3 & 1 & \\
\hline Malvaceae & Pterygota amazónica L.O.Williams ex Dorr & 1 & 3 & 1 & 3 & 3 & Anillos irregulares \\
\hline Meliceae & Cedrela odorata $\mathrm{L}$ & 1 & $1 \mathrm{y} 2$ & 3 & 1 & 1 & \\
\hline Meliceae & Guarea guidonia (L.) Sleumer & 3 & 3 & 3 & 3 & 3 & Anillos indistintos (no visibles) \\
\hline Meliceae & Guarea macrophylla Vahl. & 3 & 3 & 3 & 3 & 3 & Anillos indistintos (no visibles) \\
\hline Moraceae & Brosimum alicastrum $\mathrm{Sw}$. & 1 & 3 & 1 & 3 & 1 & \\
\hline Moraceae & Clarisia racemosa Ruiz \& Pav. & 3 & 3 & 3 & 2 & 31 & Parénquima en bandas anchas \\
\hline Moraceae & Ficus lauretana Vázq. Avila & 3 & 3 & 3 & 2 & 31 & Parénquima en bandas anchas \\
\hline Moraceae & Brosimum multinervium C.C. Berg & 1 & 3 & 2 & 3 & 1 & \\
\hline Moraceae & Ficus insipida Willd. & 2 & 3 & 3 & 2 & 31 & Parénquima en bandas anchas \\
\hline Moraceae & Poulsenia armata (Miq.) Standl & 3 & 3 & 3 & 3 & 3 & Anillos indistintos (no visibles) \\
\hline Moraceae & Pseudolmedia laevigata Trecul. & 3 & 3 & 3 & 2 & 3 & Anillos irregulares \\
\hline Moraceae & Pseudolmedia laevis (Ruiz \& Pavon) J.F. Macbr & 2 & 3 & 3 & 2 & 31 & Parénquima en bandas anchas \\
\hline Moraceae & Pseudolmedia rigida (K1. \& Karst.) Cuatr. & 1 & 3 & 1 & 2 & 1 & \\
\hline Myristicaceae & $\begin{array}{l}\text { Otoba glycicarpa (Ducke) W. Rodriguez \& T.S. } \\
\text { Jaramillo }\end{array}$ & 3 & 3 & 3 & 3 & 3 & Anillos indistintos (no visibles) \\
\hline Myristicaceae & Otoba parvifolia (Markgr.) A.H. Gentry & 2 & 3 & 2 & 3 & 27 & Transición gradual de zonas fibrosas \\
\hline Myristicaceae & Virola calophylla (Spruce) War. & 1 & 3 & 2 & 3 & 31 & Presenta anillos falsos \\
\hline Myristicaceae & Virola calophylla Var. calophylla & 1 & 3 & 2 & 3 & $3 \mathrm{I}$ & Presenta anillos falsos \\
\hline Myristicaceae & Virola duckei A.C. S.M. & 3 & 3 & 2 & 3 & 3 & Anillos indistintos (no visibles) \\
\hline Myristicaceae & Virola flexuosa A.C.Sm. & 2 & 3 & 1 & 3 & 21 & Presenta anillos falsos \\
\hline Myrtaceae & Myrcianthes rhopaloides (Kunth) McVagh & 3 & 3 & 3 & 3 & 3 & Anillos irregulares \\
\hline Podocarpaceae & Retrophyllum rospigliosii (Pilg) C.N.Page & 3 & - & 2 & 3 & 3 & Anillos estrechos \\
\hline Phyllantaceae & Hieronyma macrocarpa Müll. Arg. & 3 & 3 & 3 & 3 & 3 & Anillos indistintos (no visibles) \\
\hline Proteaceae & Roupala montana Aubl. & 3 & 3 & 3 & 3 & $3 \mathrm{I}$ & $\begin{array}{l}\text { Radios multiseriados de hasta } 30 \text { células } \\
\text { de ancho }\end{array}$ \\
\hline Rosaceae & Prunus detrita J.F. Macbr. & 2 & 3 & 2 & 3 & 27 & Transición gradual de zonas fibrosas \\
\hline Rhizophoraceae & Sterigmapetalum obovatum Kuhlm & 2 & 3 & 3 & 2 & 31 & Parénquima en bandas anchas \\
\hline Rubiaceae & $\begin{array}{l}\text { Calycophyllum spruceaunum (benth.) Hookf. } \\
\text { exSchum }\end{array}$ & 1 & 3 & 1 & 3 & 1 & \\
\hline Rubiaceae & $\begin{array}{l}\text { Calycophyllum megistocaulum (K.Krause) } \\
\text { C.M.Taylor }\end{array}$ & 2 & 3 & 2 & 3 & 27 & Transición gradual de zonas fibrosas \\
\hline Rubiaceae & Macrocnemum roseum (Ruiz \& Pav.) Wedd. & 3 & 3 & 3 & 3 & 3 & Anillos irregulares \\
\hline Sapotaceae & Pouteria guianensisAubl & 2 & 3 & 2 & 3 & 27 & Transición gradual de zonas fibrosas \\
\hline Sapotaceae & Chrysophyllum prieurii A. DC. & 2 & 3 & 2 & 2 & 27 & Transición gradual de zonas fibrosas \\
\hline Staphyleaceae & Huertea glandulosa (Ruiz \& Pavon) Steud & 2 & 3 & 2 & 3 & 27 & Transición gradual de zonas fibrosas \\
\hline Theaceae & Gordonia fruticosa (Schrad.) H. Keng & 2 & 3 & 3 & 3 & 2 & Anillos poco distintos en partes del leño \\
\hline Urticaceae & Cecropia membranaceae Trécul & 3 & 3 & 3 & 3 & 35 & Anillos indistintos (no visibles) \\
\hline Urticaceae & Coussapoa ovalifolia Trécul & 2 & 3 & 3 & 2 & $3 \mathrm{I}$ & Parénquima en bandas anchas \\
\hline Urticaceae & Pourouma guianensis Aubl. & 2 & 3 & 1 & 2 & 3 & Anillos poco distintos en partes del leño \\
\hline Vochysiaceae & Vochysia vismifolia Spruce \& Warm. & 2 & 3 & 2 & 3 & 27 & Transición gradual de zonas fibrosas \\
\hline
\end{tabular}


muchos factores, entre ellos las condiciones bióticas y abióticas del ambiente que rodea al individuo, así como también al aspecto genético de la especie.

Retrophyllum rospigliosii (Pilg) C.N. Page, es la única conífera de las 80 especies estudiadas que muestra los anillos de crecimiento indistintos macroscópicamente, característica respaldada por León \& Espinoza (2001), quienes mencionan que las células de las coníferas forman madera temprana con células de paredes delgadas y lúmenes amplios, que otorgan a esta parte del crecimiento un tono más claro que el que tiene la zona con madera tardía, y la transición entre una zona y otra puede ser gradual o abrupta. En esta especie la transición es gradual por ello no se diferencian los anillos de crecimiento macroscópicamente.

Con respecto a la variación de diámetros de los vasos de las especies estudiadas se observó que 12 especies presentan diferencias estadísticas significativas para el diámetro de los vasos, de los cuales 11 mostraron diferencias altamente significativas. Así mismo López (2006), menciona que la combinación de diámetros grandes y longitudes cortas de los vasos en madera temprana tiene una estrecha relación con la eficiencia en transporte del agua, mientras que los diámetros pequeños y longitudes grandes en madera tardía tienen como función principal evitar la ocurrencia de embolismos durante el periodo de sequía. Así también, León \& Espinoza (2001), documentan que a medida que aumenta el tamaño de los poros se produce un incremento en la eficiencia de la conducción del agua. Estos resultados podrían sugerir que el crecimiento de estas especies responde a la variación de la precipitación existente en la zona de estudio.

En lo que referente a la diferencia del diámetro de fibras de madera temprana y tardía se aprecia que 10 especies presentaron diferencias estadísticas, de las cuales ocho reportaron diferencias estadísticas altamente significativas. $\mathrm{Al}$ respecto, Díaz (2003) menciona que las diferencias de dimensiones de las células en estas dos partes del anillo (madera temprana y madera tardía) producen diferencias de tonalidad.
Por ello, en muchas especies, la madera temprana es más clara que la madera tardía.

\section{AGRADECIMIENTOS}

Al proyecto "Determinación de Incrementos Anuales y Turnos Biológicos de Rotación de Especies Forestales Tropicales para Mejorar el Aprovechamiento de Madera Comercial" del Programa de Ciencia y Tecnología, según Resolución No 220-PCM/FINCyT", formulado por Jimmy Edilson Requena Rojas y Mariano Morales, por brindarnos los medios para la ejecución de esta investigación. Al coordinador del proyecto Julio Álvarez Orellana. A Isidora Gonzales Casimiro, por su apoyo y por haber sido nuestra guía durante el desarrollo de esta investigación. A Víctor Rondón Lazo, Gerente General de la Empresa Industrial Maderera "Los Ángeles" S.A.C, Mazamari; a Uldarico Gaspar, Gerente General de la Empresa Orient Trade S.A.C., Mazamari; a Fernando Travi F. Gerente General de la Empresa NEMATSA SRL., Satipo; a Alfredo Álvarez Gerente General de la Empresa San Ramón S.A.C, Satipo; a Javier Hurtado Gerente General de la Empresa Industrial Maderera Hurtado S.A.C, Satipo; a Gustavo Alcázar Serna, Gerente General de la Empresa Maderera G y G Alcázar, Satipo; A Jaime Velásquez, Gerente General de la Empresa Maderas de Exportación S.A. MADEXSA, Satipo; por su apoyo en la recolección de las muestras en campo.

\section{RESUMEN}

El conocimiento acerca de la existencia de anillos anuales en árboles tropicales, se conoce desde principios del siglo pasado, pero fue ignorado por muchos científicos durante largo tiempo. La investigación fue realizada en el Laboratorio de Tecnología de la Madera e Industrias Forestales de la Universidad Nacional del Centro del Perú, con la finalidad de caracterizar anatómicamente los anillos de crecimiento de 80 especies arbóreas potenciales para estudios dendrocronológicos; provenientes de las provincias de Satipo y Chanchamayo en la Selva Central del Perú. Para el estudio se consideraron las normas COPANT 30:1-019, Normas Técnicas Peruanas (NTP) 251-008 y la Lista Estándar de la IAWA 1989. Entre los resultados más sobresalientes tenemos: el $30 \%$ de las ochenta especies 
estudiadas tienen buen potencial para estudios dendrocronológicos. La mayoría de estas especies, están delimitadas por una banda de fibras acortadas radialmente. Entre los problemas encontrados, se puede destacar la presencia de anillos con dificultad para ser visualizados, la presencia de anillos irregulares y la presencia de parénquima en bandas. Las características microscópicas, muestran que la variación significativa en dimensiones de fibras y vasos entre zonas de crecimiento (madera temprana y madera tardía), de las especies potenciales para dendrocronología, tienen una producción de células en forma periódica, lo que podría sugerir la formación anual de cada anillo.

Palabras claves: características anatómicas, anillos de crecimiento, madera temprana, madera tardía, dendroconología.

\section{REFERENCIAS}

Campos, L. 2009. Dendrocronología en árboles de Tornillo, Cedrelinga cateniformis Ducke (Fabaceae), del Centro de Investigaciones Jenaro Herrera en el noreste de la Amazonia, Región Loreto-Perú. Tesis Ingeniería Forestal, Universidad Nacional Agraria La Molina, Lima, Perú.

Carlquist, S. 1988. Comparative Wood anatomy, systematic, ecological and evolutionary aspects of dicotyledonous wood. Springer-Verlag, Berlín, Alemania.

Chavesta, C. 2006. Separata de Capacitación Sobre Identificación de maderas. Universidad Nacional Agraria La Molina, USAID, Lima, Perú.

Díaz, O. 2003. Anatomía de Maderas. Universidad Austral de Chile, Valdivia, Chile.

IAWA. 1989. List of microscopic features for hard wood identification. International association of wood and atomists. IAWA Bulletin n.s., E.A.Wheeler, P. Baas \& P.E. Gasson. Editorial Board, Leyden, Holland.

Instituto de investigaciones de la Amazonia Peruana (IIAP). 2011. Zonificación ecológica y económica de la provincial de Satipo. Lima, Perú.
León, W. \& N. Espinoza. 2001. Anatomía de la Madera. Consejo de Publicaciones, Mérida, Venezuela.

López, A., J. Valdez \& R. Terrazas. 2006. Anillos de Crecimiento y su Periodicidad en Tres Especies Tropicales del Estado de Colima, México. Red de Revistas Científicas de América Latina y el Caribe, España y Portugal 4: 533-544.

López, L., R. Villalba \& C. Peña. 2002. Periodicidad en el crecimiento de siete árboles forestales, usando como indicador cicatrices de fuego, marcados durante el incendio forestal de 1995, ocurrido en la Chonta, Guarayos Santa Cruz, Bolivia. IANIGLA/CONICET. 330: 1-27.

Mendoza, B. 1993. Conceptos Básicos de Manejo Forestal. Editorial UTEHA, D.F., México.

Návar, J., J. Nájera \& E. Jurado. 2001. Preliminary Estimates of Biomass Growth in the Tamaulipan Thornscrub of Northeastern Mexico. J. Arid Environ. 47: 281-290.

Tomazello, M., C. Lisi, N. Hansen \& G. Cury. 2004. Anatomical features of increment zones in different tree species in the State of Sao Paulo, Brazil. Sci. Forestalis 66: 46-55.

Vera, A., D. Ortega, P. Pérez, J. Rosero \& M. Chavesta. 2011. Avances en la caracterización e identificación anatómica de los anillos de crecimiento de la especie Junglans neotropica Diels (Nogal) para estudios dendrocronológicos en el fundo La Génova, Junín. Rev. Xilema 24: 5-11.

Young, K. \& B. León. 1999. Peru`s sumid eastern montane forest: an overview of their physical setting, biological diversity, human use and settlement, and conservation needs. Centre for Research on the Cultural and Biological Diversity of Andean Rainforests (DIVA) Technical Report 5: 1-97.

Worbes, M. 1989. Growth rings, increment and age of trees in inundation forest, savannas and a mountain forest in the neotropics. IAWA Bulletin new series 10: 109-122. 
